\title{
MadEvent: Automatic event generation with MadGraph*
}

\author{
Fabio Maltoni and Tim Stelzer \\ Department of Physics \\ University of Illinois at Urbana-Champaign \\ 1110 West Green Street \\ Urbana, IL 61801 \\ E-mail: tstelzer@uiuc.edu \\ E-mail: maltoni@uiuc.edu
}

\begin{abstract}
We present a new multi-channel integration method and its implementation in the multi-purpose event generator MadEvent, which is based on MadGraph. Given a process, MadGraph automatically identifies all the relevant subprocesses, generates both the amplitudes and the mappings needed for an efficient integration over the phase space, and passes them to MadEvent. As a result, a process-specific, stand-alone code is produced that allows the user to calculate cross sections and produce unweighted events in a standard output format. Several examples are given for processes that are relevant for physics studies at present and forthcoming colliders.
\end{abstract}

*http://madgraph.physics.uiuc.edu 


\section{Contents}

1. Introduction 1

2. Single-Diagram-Enhanced multi-channel integration 3

3. MadEvent at work: some examples 5

4. Conclusions and Outlook 6

\section{Introduction}

The high energy and luminosity of the present and future colliders, from the Tevatron to the Large Hadron Collider to the proposed $500 \mathrm{GeV}$ Linear Collider, will offer the widest range of physics opportunities for the exploration of the high-energy frontier. Among the highest priorities is understanding not only the nature of the electroweak symmetry breaking but also the mechanism through which the electroweak scale stabilizes. Simple and very robust arguments indicate that this scale should be less than one $\mathrm{TeV}$, very much in the reach of the above mentioned colliders. At this energy, multijet final states in association with leptons or missing energy constitutes the most interesting data samples. The quest for the Higgs boson(s) and/or for supersymmetric particles will rely on our ability to predict and understand the standard-model processes which are the backgrounds to these searches. In general, not only pure QCD interactions lead to multijet configurations, but also the production of vector bosons or top quarks that subsequently decay into quarks yield similar signatures with many particles in the final state.

Although straightforward in principle, the evaluation of the cross sections and especially the generation of unweighted events for such processes is not a trivial task. First of all, the number of subprocesses contributing to a process can be fairly large. For instance, for $W+4$-jet production in $p p$ collisions there are 498 distinct partonparton subprocesses. Clearly, it is desirable to automate not only the identification of these subprocesses, but also their integration and event generation.

Unfortunately, the complexity of each subprocess also grows quickly with the number of particles in the final state. If the amplitudes involve identical particles then the growth is factorial. A typical example, amply discussed in the literature [1, 2, [3], is the computation of $n$-gluon amplitudes where already for a relatively small number 
of gluons produced, say $n \gtrsim 6$, clever reorganizations of the perturbative expansion have to be employed to reduce the factorial growth to a more manageable exponential

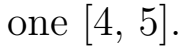

The second step is the integration of the squared amplitude over the phase space, which is necessarily performed using Monte Carlo techniques. This task involves solving several issues. In the first place, the amplitude results in a very complicated function of the momenta which displays sharp peaks in many region of the phase space. In order to achieve an efficient numerical integration, one needs to identify the position of these peaks and to map them onto several different sets of variables, which are usually referred to as "channels". In principle, both tasks are not trivial. Fortunately, a standard Feynman diagram expansion provides the necessary information on the structure (position and shape) of the peaks in the matrix elements. In general, the main source of variation of the amplitude in a given region of the phase space can be associated with one (or more) of the propagators in one (or more) diagrams becoming large. Typically, this happens either when collinear-divergent limits are approached or when virtual massive particles, with a small width, are close to their mass-shell.

At present, the available codes for the automated generation of phase space are based on identifying singularities in Feynman diagrams [6, 7]. As we will show in the following, we use a similar approach for generating the phase-space mappings. However, our method differs from the previous ones in that we propose to exploit the information gathered from the Feynman diagrams to reorganize and simplify the way we perform the integration over the various channels.

In this paper we present a new multi-channel integration method, which we will call Single-Diagram-Enhanced, and its implementation in the new multi-purpose event generator MadEvent which is powered by a new version of MadGraph [8]. Given a user process, MadGraph automatically generates the amplitudes for all the relevant subprocesses and produces the mappings for the integration over the phase space. This process-dependent information is passed to MadEvent, and a stand-alone code is produced that allows the user to calculate cross sections and to obtain unweighted events. Once the events have been generated (and all the necessary information stored $^{1}$ ) they may be passed to any shower Monte Carlo program (such as HERWIG [10] or PYTHIA [11, 12]) where partons are perturbatevely evolved through the emission of QCD radiation, and eventually turned into physical states (hadronization).

This paper is organized as follows. In the next section we present our method for mapping the phase space and integrating the squared amplitude. In Section 3, we provide some examples of the results that can be obtained with MadEvent. The conclusions and a brief discussion of the outlook are addressed in the last section.

\footnotetext{
${ }^{1}$ In addition to the usual kinematic quantities, such as masses and momenta, the spin, the color connection, and the flavour of each of the final-state partons may be needed for each event. This information is stored in the "Les Houches" format [9.
} 


\section{Single-Diagram-Enhanced multi-channel integration}

In this section we present our approach for integrating a generic squared amplitude over the phase space of the final-state particles. We first review the usual multichannel integration method, following closely Ref. [13], since this serves to establish the notation and allows us to point out the issues relevant to the subsequent discussion.

Let us consider $f(\vec{\Phi})$ the function to be integrated over the set of phase-space variables $\vec{\Phi}$. Given $N$ the number of particles in the final state, $\vec{\Phi}$ is of dimension $d=3 N-4$. In order to account for the complex structure of $f$ several mappings of the Monte Carlo variables $\vec{x} \in[0,1]^{d}$ are introduced and each of them generates a different probability density, $g_{i}(\vec{\Phi}), i=1, \ldots, n$, where $n$ is the total number of channels. ${ }^{2}$ The optimization of the relative importance of the different channels is achieved by introducing a set of weights $\alpha_{i}, \sum_{i=1}^{n} \alpha_{i}=1, \alpha_{i} \geq 0$, and defining a total probability density $g(\vec{\Phi})=\sum_{i=1}^{n} \alpha_{i} g_{i}(\vec{\Phi})$, which combines the weights of each channel and is still correctly normalized. The weight of a phase space point $\vec{\Phi}$ can be written as $w(\vec{\Phi})=f(\vec{\Phi}) / g(\vec{\Phi})$ and the result of the Monte Carlo integration and its variance are:

$$
\begin{gathered}
I=\int d \vec{\Phi} g(\vec{\Phi}) w(\vec{\Phi})=\int d \vec{\Phi} f(\vec{\Phi}), \\
V(\alpha)=\int d \vec{\Phi} g(\vec{\Phi}) w(\vec{\Phi})^{2}=\int d \vec{\Phi} \frac{f(\vec{\Phi})^{2}}{g(\vec{\Phi})} .
\end{gathered}
$$

As made explicit in the notation, the result of the integration does not depend on the choice of the $\alpha_{i}$ (which can even differ iteration by iteration), while the variance does. The aim of the multi-channel approach is to find the best set of $\alpha_{i}$ which minimizes the variance $V$. Several strategies can be followed [13]. Here, we just comment on a few important aspects of this approach.

In the first place we note that for each point in the phase space, independently from the actual channel chosen, the determination of $g(\vec{\Phi})$ implies the computation of the contributions from all channels. As long as the number of channels is not very large and/or the computational burden for calculating $g(\vec{\Phi})$ is much smaller than that for $f(\vec{\Phi})$, this might not be a serious problem. However, in general, this procedure is inefficient. During the integration, the relative weight of each channel changes due to the optimization of the $\alpha_{i}$. In particular, even if some of the channels become less and less important compared to others, the time spent to calculate their contributions remains the same. Another point of weakness is that, even if the evolution of each of the $\alpha_{i}$ can be chosen to be independent from the others, in practice the $\alpha_{i}$ are

\footnotetext{
${ }^{2}$ Each probability density $g_{i}(\vec{\Phi})$ is related to the mapping through $\vec{\Phi}=G_{i}^{-1}(\vec{x})$, where $G_{i}(\vec{\Phi})$ is the primitive of $g_{i}(\vec{\Phi})$.
} 
correlated. Predicting the behavior and/or the stability of an integration with a large number of channels is very difficult and jeopardizes the robustness of the solution. ${ }^{3}$

We argue that there is a natural way to overcome the above limitations and to calculate the integral of a squared amplitude over the phase space in an efficient way. The idea is very simple and is based on the following observation. Assume that the function to be integrated could be written in terms of a basis of $n$ functions $f_{i}$ such that:

$$
f=\sum_{i=1}^{n} f_{i} \quad \text { with } \quad f_{i} \geq 0, \quad \forall i,
$$

and such that the peak structure of each $f_{i}$ can be efficiently mapped by a single channel $g_{i}$. Then, the integration of $f$ reduces to:

$$
I=\int d \vec{\Phi} f(\vec{\Phi})=\sum_{i=1}^{n} \int d \vec{\Phi} g_{i}(\vec{\Phi}) \frac{f_{i}(\vec{\Phi})}{g_{i}(\vec{\Phi})}=\sum_{i=1}^{n} I_{i}
$$

i.e., to a sum of $n$ independent integrations. For a generic integration problem, the identification of such a basis might be too difficult and the above method be simply not viable. However, in the case at hand, a natural decomposition arises from the physical content of the process. We propose to use the following basis:

$$
f_{i}=\frac{\left|A_{i}\right|^{2}}{\sum_{i}\left|A_{i}\right|^{2}}\left|A_{\mathrm{tot}}\right|^{2}
$$

where $A_{i}$ is the amplitude corresponding to a single Feynman diagram and $A_{\text {tot }}=$ $\sum_{i} A_{i}$ is the total amplitude. It is clear that each $f_{i}$ in Eq. (2.5) satisfies the conditions (2.3) and form a "complete" basis. ${ }^{4}$ More importantly, the peak structure of each $f_{i}$ is the same as of the single squared amplitude $\left|A_{i}\right|^{2}$. Finding the suitable mapping $g_{i}$ is therefore straightforward, since it can be derived from the propagator structure of the corresponding Feynman diagram. We will refer to the above algorithm as Single-Diagram-Enhanced multi-channel integration.

Decomposing the integration of the amplitude into $n$ independent integrations has immediate advantages. First, in contrast to the standard multi-channel integration, the evaluation of the weight in one channel does not require the computation of the others. This entails that, from the statistics point of view, in the Single-DiagramEnhanced integration method the complexity of the computation does not increase with the number of channels.

Second, the Single-Diagram-Enhanced integration is parallel in nature. Integrations in each channel can be performed by using different resources and the results

\footnotetext{
${ }^{3}$ This aspect can in practice hamper the possibility of using many channels if the mapping $g_{i}$ are obtained by a stratified Monte Carlo method.

${ }^{4} \mathrm{Eq}$. (2.5) is analogous to the procedure suggested by Odagiri 14 to extract the leading color behaviour of QCD amplitudes.
} 
combined only at the very end. Considering the latest developments in this field, such as the availability of PC farms at low cost, we reckon this as an alluring property of this approach.

A third useful aspect of our method is that, given a target accuracy of the integration, it is trivial to reweight the channels so that those whose contributions to the total result is small (large) are evaluated with a smaller (greater) number of Monte Carlo points. One simply introduces the analogues of the $\alpha_{i}$ as in a standard multi-channel method, defining them as $\alpha_{i}=I_{i} / I$. This realizes in practice the desirable property that the computational time spent on a single channel should be proportional to its relevance to the final result.

Another interesting property of the decomposition (2.5) is its intrinsic modularity. The number of the diagrams to be included in the decomposition is completely arbitrary and dictated only by convenience. In general, the number of channels will be less than the number of Feynman diagrams. The first important example is when $k$ identical particles are present in the final state and $n$ can be easily reduced by a factor $k$ !. Another case is when some diagrams do not display any peaked behavior and are dropped from Eq. (2.5). Alternately, some diagrams can be efficiently grouped together if the form of the resulting $g_{i}$ is predicted by general arguments, such as radiation coherence.

\section{MadEvent at work: some examples}

In this section, we present some results for processes relevant at hadron colliders, which have been obtained with MadEvent. The purpose is to show the versatility and the potential of our approach and also to provide benchmark cross sections for the user. Detailed information on how to use the MadGraph/MadEvent package together with a web interface for generating the code for a given process is available at http://madgraph.physics.uiuc.edu. In order to facilitate the comparison with other available codes we have chosen a very simple set of cuts and conventions, which are summarized in Table 6 . In particular, the cuts in transverse energy and $\Delta R$ are left quite loose so that regions of the phase space where the matrix elements peak are not left out and the efficiency of our integration technique can be fully tested. ${ }^{5}$

In Table 2 we present the cross sections involving the production one or more vector bosons in association with jets, both at the Tevatron and LHC. Apart from the interest they might have on their own, these processes contribute to some of the most important backgrounds for top-quark studies $(W+$ jets, $W b \bar{b}+$ jets $)$, for the Higgs searches $\left(W^{+} W^{-}+\right.$jets, $W b \bar{b}+$ jets, $Z Z+$ jets) and for SUSY particles searches $(Z+$ jets, with $Z \rightarrow \nu \bar{\nu})$. In the single vector boson production, final states are

\footnotetext{
${ }^{5}$ In fact, at the LHC such cuts are not sufficient to ensure a good behaviour of the perturbative series for some of the cross sections shown, as it is apparent by comparing the processes with $n$ jets with the corresponding ones with $(n+1)$ jets.
} 
leptons and so that the vector bosons are not contrained to be on-shell. This entails, for instance, that cross sections involving a $Z$ boson, correctly include the diagrams where $\gamma^{*} \rightarrow \ell^{+} \ell^{-}$and their interference with $Z^{*} \rightarrow \ell^{+} \ell^{-}$.

For simplicity, in the case of double vector boson production, we present the results where the vector bosons are produced on shell and are not decayed. Nonetheless, we stress that MadEvent correctly handles also the decays, where non-trivial phase-space mappings have to be introduced. An example is provided by $W^{+} W^{-}$ production where contributions from diagrams where $\gamma^{*} / Z \rightarrow W^{+} W^{-} \rightarrow \ell^{+} \ell^{-} \nu \bar{\nu}$ appear, which demand particular care. In this case, there are different regions in the phase space which can give large contributions, coming from the $Z$ and one of the $W$ 's, or both $W$ 's being on shell. We have ensured that the procedure used for the mapping of the phase space automatically accounts for all the possibilities, so that no approximation is introduced.

When possible, we have compared our results with the ones obtained by running available codes (such as VECBOS [15], Wbbgen [16] and MCFM [17]) and found good agreement. We plan to conduct a detailed set of comparisons with the recently released Alpgen [18] in the future.

Table 3 contains the cross sections for the production one or more heavy-quark pairs in association with jets, both at the Tevatron and LHC. In the case of $t \bar{t} b \bar{b}$ production we also give the cross sections at order $\alpha_{\mathrm{em}}^{2} \alpha_{S}^{2}$. These last ones constitute the irreducible backgrounds in the search of the Higgs when it is produced in association with a top-quark pair. Also, the cross section for two bottom-quark pairs in association with jets are listed. In the case of two bottom-quark pairs we have also performed a detailed comparison with the results of Ref. [19] and found good agreement.

In Table 1 cross sections are given for the production of an Higgs boson in association with a top-quark pair, with jets ("W-boson fusion") and in association with a $W$ boson, both at the Tevatron and LHC.

\section{Conclusions and Outlook}

In this paper we have introduced a new integration method, called Single-DiagramEnhanced multi-channel integration, and its implementation in the multi-purpose event generator MadEvent.

We have discussed how the problem of integrating over the phase space amplitudes that involve a rather large number of particles in the final state can be reduced to the integration of amplitudes associated to single Feynman diagrams. The latter are much simpler tasks and can be tackled by many of the techniques already present in the literature. Our method best applies to the automated computation of cross sections (and, more importantly, to the generation of unweighted events) for 


\begin{tabular}{|c|c|}
\hline colliders & $\begin{array}{l}\text { Tevatron: } p \bar{p} @ 2 \mathrm{TeV} \\
\text { LHC: } p p @ 14 \mathrm{TeV}\end{array}$ \\
\hline QCD and EW couplings and parameters & $\begin{array}{l}\alpha_{S}\left(M_{Z}\right)=0.1185 \\
\alpha_{\mathrm{em}}\left(M_{Z}\right)=1 / 128.9, \sin ^{2} \theta_{W}=0.2312 \\
\text { CKM matrix: diagonal }\end{array}$ \\
\hline p.d.f. & CTEQ5L (parametric) \\
\hline scales & $\mu_{F}=\mu_{R}=M_{Z}$ \\
\hline vector boson masses and widths & $\begin{array}{l}m_{Z}=91.188 \mathrm{GeV}, \Gamma_{Z}=2.495 \mathrm{GeV} \\
m_{W}=79.96 \mathrm{GeV}, \Gamma_{W}=2.06 \mathrm{GeV}\end{array}$ \\
\hline heavy-quark (pole) masses & $m_{t}=174.3 \mathrm{GeV}, m_{b}=4.7 \mathrm{GeV}$ \\
\hline Higgs parameters and couplings ${ }^{6}$ & $\begin{array}{l}M_{H}=120 \mathrm{GeV}, \Gamma_{H}=3.7 \cdot 10^{-3} \mathrm{GeV} \\
y_{t}=0.97, y_{b}=0.017\end{array}$ \\
\hline kinematic cuts & $\begin{array}{l}E^{T}>10 \mathrm{GeV},|\eta|<2.5, \Delta R>0.4, \\
\text { for all the final-state particles }\end{array}$ \\
\hline
\end{tabular}

Table 1: Couplings and conventions used in the calculation of the benchmark cross sections.

processes where many different parton subprocess contribute, such as vector boson production in association with jets at hadron colliders and many others.

We have presented some applications of MadEvent, a multi-purpose event generator based on the above ideas, to studies relevant at the present and forthcoming colliders. All the necessary steps to generate a set of unweighted events are performed automatically. The user only inputs the process and the desired integrated luminosity or the total number of unweighted events.

Although in principle there is no limitation to the number of particles that can be considered in the final state, in practice this still depends on the complexity of the process itself and on the total number of Feynman diagrams involved. Currently, the package is limited to ten thousands diagrams per subprocess. So, for example, $W+5$ jets is feasible but close to the present limit.

Several improvements that will extend the ability of MadGraph to deal with a larger number of QCD partons are under study. One necessary step is the "factorization" of the amplitude in order to reduce the complexity of the calculation from a factorial growth to an exponential one, as already achieved for non-QCD amplitudes in Refs. [20, 21, 22 and extended to the QCD amplitudes in Refs. [4, 20, 18]. In the latter case, a reorganization of the color structure is also needed and the sum over the colors of the external QCD partons can be efficiently performed by a Monte Carlo integration. Work in this direction is in progress [23].

\footnotetext{
${ }^{6}$ In fact $\Gamma_{H}$ and $y_{b}$ do not enter the results presented here, but we prefer to leave them as a reference for the user.
} 


\section{Acknowledgments}

We thank Scott Willenbrock and Michelangelo Mangano for useful discussions. We are also grateful to Henry Frisch, Bruce Knuteson for their encouragement and Uli Baur and John Campbell for their help in testing the package.

\section{References}

[1] F. A. Berends and W. T. Giele, Recursive calculations for processes with $n$ gluons, Nucl. Phys. B306 (1988) 759.

[2] F. A. Berends, W. T. Giele, and H. Kuijf, On six jet production at hadron colliders, Phys. Lett. B232 (1989) 266.

[3] M. L. Mangano and S. J. Parke, Multiparton amplitudes in gauge theories, Phys. Rept. 200 (1991) 301-367.

[4] P. Draggiotis, R. H. P. Kleiss, and C. G. Papadopoulos, On the computation of multigluon amplitudes, Phys. Lett. B439 (1998) 157-164, http://arXiv.org/abs/hep-ph/9807207.

[5] F. Caravaglios, M. L. Mangano, M. Moretti, and R. Pittau, A new approach to multi-jet calculations in hadron collisions, Nucl. Phys. B539 (1999) 215-232, http://arXiv.org/abs/hep-ph/9807570.

[6] C. G. Papadopoulos, Phegas: A phase space generator for automatic cross-section computation, Comput. Phys. Commun. 137 (2001) 247-254, http://arXiv.org/abs/hep-ph/0007335.

[7] F. Krauss, R. Kuhn, and G. Soff, Amegic ++ 1.0: A matrix element generator in $c++$, JHEP 02 (2002) 044, http://arXiv.org/abs/hep-ph/0109036.

[8] T. Stelzer and W. F. Long, Automatic generation of tree level helicity amplitudes, Comput. Phys. Commun. 81 (1994) 357-371, http://arXiv.org/abs/hep-ph/9401258].

[9] E. Boos et. al., Generic user process interface for event generators, http://arXiv.org/abs/hep-ph/0109068.

[10] G. Marchesini and B. R. Webber, Monte carlo simulation of general hard processes with coherent qcd radiation, Nucl. Phys. B310 (1988) 461.

[11] H.-U. Bengtsson and T. Sjostrand, The lund monte carlo for hadronic processes: Pythia version 4.8, Comput. Phys. Commun. 46 (1987) 43.

[12] T. Sjostrand, High-energy physics event generation with pythia 5.7 and jetset 7.4, Comput. Phys. Commun. 82 (1994) 74-90. 
[13] R. Kleiss and R. Pittau, Weight optimization in multichannel monte carlo, Comput. Phys. Commun. 83 (1994) 141-146, http://arXiv.org/abs/hep-ph/9405257.

[14] K. Odagiri, Color connection structure of (supersymmetric) QCD (2 $\rightarrow$ 2) processes, JHEP 10 (1998) 006, http://arXiv.org/abs/hep-ph/9806531].

[15] F. A. Berends, H. Kuijf, B. Tausk, and W. T. Giele, On the production of a w and jets at hadron colliders, Nucl. Phys. B357 (1991) 32-64.

[16] M. L. Mangano, M. Moretti, and R. Pittau, Multijet matrix elements and shower evolution in hadronic collisions: $W$ b anti- $b+n$ jets as a case study, http://arXiv.org/abs/hep-ph/0108069.

[17] J. Campbell and R. K. Ellis, Next-to-leading order corrections to $w+2 j e t$ and $z+$ 2jet production at hadron colliders, Phys. Rev. D65 (2002) 113007, http://arXiv.org/abs/hep-ph/0202176.

[18] M. L. Mangano, M. Moretti, F. Piccinini, R. Pittau, and A. D. Polosa, Alpgen, a generator for hard multiparton processes in hadronic collisions, http://arXiv.org/abs/hep-ph/0206293.

[19] S. Tsuno et. al., gr@ppa $a_{4 b}$ : A four bottom quark production event generator for $p$ p / $p$ anti-p collisions, http://arXiv.org/abs/hep-ph/0204222.

[20] F. Caravaglios and M. Moretti, An algorithm to compute born scattering amplitudes without feynman graphs, Phys. Lett. B358 (1995) 332-338, http://arXiv.org/abs/hep-ph/9507237.

[21] T. Ohl, O'mega: An optimizing matrix element generator, http://arXiv.org/abs/hep-ph/0011243.

[22] A. Kanaki and C. G. Papadopoulos, Helac: A package to compute electroweak helicity amplitudes, Comput. Phys. Commun. 132 (2000) 306-315, http://arXiv.org/abs/hep-ph/0002082.

[23] F. Maltoni, K. Paul, T. Stelzer, and S. Willenbrock, in preparation, . 


\begin{tabular}{|c|c|c|c|c|c|}
\hline Process $+n$ jets & $n$ & order & unit & Tevatron & $\mathrm{LHC}$ \\
\hline \multirow{5}{*}{$e^{+} \nu_{e}\left(e^{-} \bar{\nu}_{e}\right)$} & 0 & \multirow{5}{*}{$\alpha_{\mathrm{em}}^{2} \alpha_{S}^{n}$} & \multirow{5}{*}{$\mathrm{pb}$} & 758 & $3850(3450)$ \\
\hline & 1 & & & 182 & $1700(1520)$ \\
\hline & 2 & & & 46.6 & $742(642)$ \\
\hline & 3 & & & 12.0 & $337(279)$ \\
\hline & 4 & & & 3.19 & $156(122)$ \\
\hline \multirow{5}{*}{$e^{+} e^{-}$} & 0 & \multirow{5}{*}{$\alpha_{\mathrm{em}}^{2} \alpha_{S}^{n}$} & \multirow{5}{*}{$\mathrm{pb}$} & 210 & 1000 \\
\hline & 1 & & & 46.2 & 398 \\
\hline & 2 & & & 12.6 & 179 \\
\hline & 3 & & & 3.30 & 79.0 \\
\hline & 4 & & & 0.871 & 35.1 \\
\hline \multirow{3}{*}{$e^{+} \nu_{e}\left(e^{-} \bar{\nu}_{e}\right) b \bar{b}$} & 0 & \multirow{3}{*}{$\alpha_{\mathrm{em}}^{2} \alpha_{S}^{n+2}$} & \multirow{3}{*}{$\mathrm{fb}$} & 427 & $2330(1770)$ \\
\hline & 1 & & & 195 & $2950(2330)$ \\
\hline & 2 & & & 73.1 & $2600(1980)$ \\
\hline \multirow{3}{*}{$e^{+} e^{-} b \bar{b}$} & 0 & \multirow{3}{*}{$\alpha_{\mathrm{em}}^{2} \alpha_{S}^{n+2}$} & \multirow{3}{*}{$\mathrm{fb}$} & 165 & 3880 \\
\hline & 1 & & & 79.3 & 3080 \\
\hline & 2 & & & 28.0 & 1770 \\
\hline \multirow{3}{*}{$W^{+} W^{-}$} & 0 & \multirow{3}{*}{$\alpha_{\mathrm{em}}^{2} \alpha_{S}^{n}$} & \multirow{3}{*}{$\mathrm{pb}$} & 9.28 & 46.3 \\
\hline & 1 & & & 3.84 & 37.0 \\
\hline & 2 & & & 1.23 & 25.3 \\
\hline \multirow{3}{*}{$W^{+}\left(W^{-}\right) Z$} & 0 & \multirow{3}{*}{$\alpha_{\mathrm{em}}^{2} \alpha_{S}^{n}$} & \multirow{3}{*}{$\mathrm{pb}$} & 1.49 & $10.0(7.25)$ \\
\hline & 1 & & & 0.633 & $10.7(7.31)$ \\
\hline & 2 & & & 0.209 & $9.15(6.40)$ \\
\hline \multirow{3}{*}{$Z Z$} & 0 & \multirow{3}{*}{$\alpha_{\mathrm{em}}^{2} \alpha_{S}^{n}$} & \multirow{3}{*}{$\mathrm{pb}$} & 1.04 & 6.70 \\
\hline & 1 & & & 0.440 & 4.95 \\
\hline & 2 & & & 0.133 & 2.97 \\
\hline
\end{tabular}

Table 2: Benchmark cross sections for single and double vector boson production in association with jets at hadron colliders. The decays into leptons, $W \rightarrow e \nu_{e}$ and $\gamma^{*} / Z \rightarrow$ $e^{+} e^{-}$, are included in the single vector boson cross sections and cuts, as described in the text, are applied to all the final state particles (jets and leptons). In the case of $W W, W Z, Z Z$ production, the vector bosons are not decayed and are produced on shell. Statistical errors for all processes are at the percent level. 


\begin{tabular}{|c|c|c|c|c|c|}
\hline Process $+n$ jets & $n$ & order & unit & Tevatron & $\mathrm{LHC}$ \\
\hline \multirow{4}{*}{$t \bar{t}$} & 0 & \multirow{4}{*}{$\alpha_{S}^{n+2}$} & \multirow{4}{*}{$\mathrm{pb}$} & 7.67 & 579 \\
\hline & 1 & & & 3.53 & 762 \\
\hline & 2 & & & 1.24 & 660 \\
\hline & 3 & & & 0.385 & 460 \\
\hline \multirow{4}{*}{$b \bar{b}$} & 0 & \multirow{4}{*}{$\alpha_{S}^{n+2}$} & \multirow{4}{*}{$\mathrm{nb}$} & 832 & 15000 \\
\hline & 1 & & & 115 & 3010 \\
\hline & 2 & & & 29.0 & 1110 \\
\hline & 3 & & & 6.35 & 356 \\
\hline \multirow{4}{*}{$t \bar{t} b \bar{b}$} & 0 & \multirow{2}{*}{$\alpha_{S}^{n+4}$} & \multirow{2}{*}{$\mathrm{fb}$} & 14.5 & 3890 \\
\hline & 1 & & & 8.21 & 6440 \\
\hline & 0 & \multirow{2}{*}{$\alpha_{\mathrm{em}}^{2} \alpha_{S}^{n+2}$} & \multirow{2}{*}{$\mathrm{fb}$} & 1.14 & 336 \\
\hline & 1 & & & 0.747 & 380 \\
\hline \multirow{4}{*}{$b \bar{b} b \bar{b}$} & 0 & \multirow{2}{*}{$\alpha_{S}^{n+4}$} & \multirow{2}{*}{$\mathrm{pb}$} & 86.1 & 4050 \\
\hline & 1 & & & 41.0 & 546 \\
\hline & 0 & \multirow{2}{*}{$\alpha_{\mathrm{em}}^{2} \alpha_{S}^{n+2}$} & \multirow{2}{*}{$\mathrm{fb}$} & 676 & 16100 \\
\hline & 1 & & & 428 & 4090 \\
\hline
\end{tabular}

Table 3: Benchmark cross sections for heavy-quark pair production in association with jets (light quarks and gluons) at hadron colliders. The heavy quarks are produced on shell and are not decayed. In $t \bar{t} b \bar{b}$ and $b \bar{b} b \bar{b}$ production, contributions from the exchange of a virtual Higgs have been excluded. Cuts, as described in the text, are applied to all the final state particles. Statistical errors for all processes are at the percent level.

\begin{tabular}{c|c|c|c|c|c}
\hline \hline Process $+n$ jets & $n$ & order & unit & Tevatron & LHC \\
\hline \multirow{2}{*}{$h$} & 2 & \multirow{2}{*}{$\alpha_{\mathrm{em}}^{3} \alpha_{S}^{n-2}$} & $\mathrm{fb}$ & 157 & 1550 \\
& 3 & & & 89.3 & 1000 \\
\hline \multirow{3}{*}{$t \bar{t} h$} & 0 & & & 7.30 & 545 \\
& 1 & \multirow{2}{*}{$y_{t}^{2} \alpha_{S}^{n+2}$} & $\mathrm{fb}$ & 3.14 & 830 \\
& 2 & & & 1.00 & 852 \\
\hline \multirow{3}{*}{$W^{+}\left(W^{-}\right) h$} & 0 & & & 67.9 & $563(391)$ \\
& 1 & \multirow{2}{*}{$\alpha_{\mathrm{em}}^{2} \alpha_{S}^{n}$} & $\mathrm{fb}$ & 29.3 & $425(290)$ \\
& 2 & & & 9.11 & $250(168)$ \\
& 3 & & & 2.34 & $137(89)$ \\
\hline \hline
\end{tabular}

Table 4: Benchmark cross sections for Higgs production in association with a top-quark pair, with jets (EW processes) and in association with a $W$ boson, at hadron colliders. The heavy quarks and the Higgs and the $W$ are produced on shell and are not decayed. Cuts, as described in the text, are applied to all the final state particles. Statistical errors for all processes are at the percent level. 